University of Nebraska - Lincoln

DigitalCommons@University of Nebraska - Lincoln

November 2007

\title{
Structure and thermophysical properties of single-wall Si nanotubes
}

\author{
Xinwei Wang \\ University of Nebraska-Lincoln, xwang3@unl.edu \\ Zhen Huang \\ University of Nebraska-Lincoln \\ Tao Wang \\ Zhejiang University, Hangzhou, P. R. China \\ Yuk Wai Tang \\ University of Nebraska-Lincoln \\ Xiao Cheng Zeng \\ University of Nebraska-Lincoln, xzeng1@unl.edu
}

Follow this and additional works at: https://digitalcommons.unl.edu/chemzeng

Part of the Chemistry Commons

Wang, Xinwei; Huang, Zhen; Wang, Tao; Tang, Yuk Wai; and Zeng, Xiao Cheng, "Structure and thermophysical properties of single-wall Si nanotubes" (2007). Xiao Cheng Zeng Publications. 78.

https://digitalcommons.unl.edu/chemzeng/78

This Article is brought to you for free and open access by the Published Research - Department of Chemistry at DigitalCommons@University of Nebraska - Lincoln. It has been accepted for inclusion in Xiao Cheng Zeng Publications by an authorized administrator of DigitalCommons@University of Nebraska - Lincoln. 


\section{Author's Accepted Manuscript}

Structure and thermophysical properties of singlewall Si nanotubes

Xinwei Wang, Zhen Huang, Tao Wang, Yuk Wai Tang, Xiao Cheng Zeng

$\begin{array}{ll}\text { PII: } & \text { S0921-4526(07)01303-8 } \\ \text { DOI: } & \text { doi:10.1016/j.physb.2007.11.016 } \\ \text { Reference: } & \text { PHYSB 302796 }\end{array}$

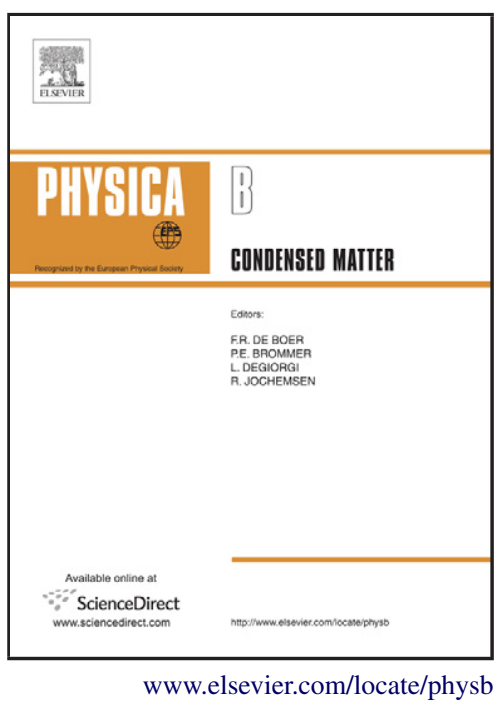

To appear in: $\quad$ Physica $B$

Received date: 5 April 2007

Revised date: 1 November 2007

Accepted date: 17 November 2007

Cite this article as: Xinwei Wang, Zhen Huang, Tao Wang, Yuk Wai Tang and Xiao Cheng Zeng, Structure and thermophysical properties of single-wall Si nanotubes, Physica B (2007), doi:10.1016/j.physb.2007.11.016

This is a PDF file of an unedited manuscript that has been accepted for publication. As a service to our customers we are providing this early version of the manuscript. The manuscript will undergo copyediting, typesetting, and review of the resulting galley proof before it is published in its final citable form. Please note that during the production process errors may be discovered which could affect the content, and all legal disclaimers that apply to the journal pertain. 


\title{
Structure and thermophysical properties of single-wall Si nanotubes
}

\author{
Xinwei Wang, ${ }^{*}$ Zhen Huang \\ Department of Mechanical Engineering, N104 Walter Scott Engineering Center \\ University of Nebraska-Lincoln, Lincoln, NE 68588-0656 \\ Tao Wang \\ State Key Laboratory of Clean Energy Utilization \\ Zhejiang University, Hangzhou, P. R. China, 310027 \\ Yuk Wai Tang, Xiao Cheng Zeng \\ Department of Chemistry, University of Nebraska-Lincoln, Lincoln, NE 68588-0304
}

\begin{abstract}
In this work, molecular dynamics (MD) simulation based on the environment-dependent interatomic potential is carried out to explore the structure, atomic energy distribution, and thermophysical properties of single-wall $\mathrm{Si}$ nanotubes (SWSNTs). The unique structure of SWSNTs leads to a wider range energy distribution than crystal $\mathrm{Si}$ (c-Si), and results in a bond order in the range of $4.8 \sim 5$. The thermal conductivity of SWSNTs is much smaller than that of bulk Si, and shows significantly slower change with their characteristic size than that of Si films. Out of the three types of SWSNTs studied in this work, pentagonal SWSNTs have the highest thermal conductivity while hexagonal SWSNTs have the lowest one. The specific heat of SWSNTs is a little larger than that of bulk c-Si. Pentagonal and hexagonal SWSNTs have close specific heats which are a little larger than that of rectangular SWSNTs.
\end{abstract}

PACS: $67.80 . \mathrm{Gb} ; 31.15 . \mathrm{Qg} ;$ 61.46.Fg

Keywords: Si nanotubes, atomic energy distribution, thermal conductivity, specific heat

${ }^{*}$ Corresponding author. Email: xwang3@unl.edu; Tel: (402) 472-3089; Fax: (402) 472-1465. 


\section{Introduction}

In the past decade, nanotechnology has been developed and applied broadly in various fields. The small scale of nanomaterials provides a lot of advantages for miniature device design and manufacture. As a result, various properties of nanomaterials have been studied. It is well documented that properties of nanomaterials can be much different from those of the bulk counterparts due to the unique structure and dimension of nanomaterials. After the discovery of carbon nanotubes (CNTs) [1], a wide spectrum of research has been carried out to study this kind of one dimensional nanostructure. It is shown the thermal conductivity of CNTs strongly depends on their length due to significant boundary scattering of energy carriers at the CNT ends [2]. Despite the similarity in the atomic structure between Si and C, Si nanotubes with a similar structure to CNTs or bulk crystal $\mathrm{Si}(\mathrm{c}-\mathrm{Si}$ ) have not been found. Recently, single-wall $\mathrm{Si}$ nanotubes (SWSNTs) with stable structure of different cross section shapes: rectangular, pentagonal and hexagonal have been predicted using atomistic simulations [3]. The local geometric structure of SWSNTs is very different from that of bulk Si and surface-passivated 1D Si nanowires. It is also different from the "hypothetical" carbon-nanotube-like SWSNTs which are assumed to be composed of $s p^{2}$ and $s p^{3}$ bonds. Moreover, the ab initio calculation showed an entirely opposite trend in bond gap change compared with 1D Si nanowires [4]. SWSNTs are more likely metallic than semiconducting. It will be of great interest to explore the unique structure of SWSNTs, which is different from that of $\mathrm{c}-\mathrm{Si}$ and to show how their unique structure affects their thermophysical properties. This knowledge is required in functionality and dependability design of SWSNT-based materials and devices in the future.

In the past, both experiments and simulations have been reported on studying the thermal 
conductivity of nanotube materials, largely CNTs. Experimentally, Hone et al. [5] measured the thermophysical properties of single wall CNTs and gave the temperature dependence of the thermal conductivity from 8 to $350 \mathrm{~K}$. Yang et al. [6] and Wang et al. [7] studied the thermal conductivity of multiwall CNTs using photothermal experiments and found the thermal conductivity of multiwall CNTs is much less than the theoretical prediction. For numerical studies, molecular dynamics (MD) simulation has been widely employed to study the thermal transport in CNTs $[2,8]$. Previous study by Chen and Volz [9] explored the thermal conductivity of another 1D structure allotrope of Si nanowires using equilibrium MD (EMD) simulation. The result showed the thermal conductivity was about two orders of magnitude smaller than that of bulk Si. In 1999, Volz and Chen [10] also used EMD to study the thermal conductivity of bulk cSi. Recent work on MD simulation of the thermal transport in c-Si includes that by Gomes et al. (for bulk Si) [11], Feng et al. [12] and Wang and Lawrence [13] (for c-Si thin films). Basically, there are two main methods in MD simulation to predict the thermal conductivity of materials. One method is EMD simulation using the Green-Kubo formula. This method has been used to study the thermophysical properties of amorphous [14] and crystalline [10] Si. The other one is nonequilibrium MD (NEMD) simulation which involves establishing a temperature gradient in the material. Using this method, the thermal conductivity of a two-dimensional crystal [15] and vitreous silica has been studied and the results are in good agreement with the experimental data [16].

Each method reviewed above has its own advantages and drawbacks. Work by Schelling et al. [17] shows that the simulation time is a more significant consideration for the Green-Kubo method, where very slow convergence of the heat current correlation function is observed. The 
NEMD method can greatly reduce the computational cost to obtain the same statistical errors as the EMD method. Especially in systems with very small finite-size effect due to the very small mean free path of phonons, rather small systems sizes are required by the NEMD method. The NEMD method is more suitable to study interfacial effects. The drawback of NEMD is that the use of large temperature gradients may introduce significant nonlinear effects.

In this work, NEMD simulation is used to explore the thermal conductivity of SWSNTs and EMD simulation is used to study the specific heat of SWSNTs. In addition to thermophysical properties, the structure and energy distribution of atoms in SWSNTs are also investigated to obtain better ideas about how and to what extent the structure of SWSNTs affects their thermophysical properties. Section 2 of this paper provides a brief introduction to the MD simulation method used in this work. Section 3 discusses the calculation results for SWSNTs and the comparison with crystal $\mathrm{Si}(\mathrm{c}-\mathrm{Si})$ thin films.

\section{Methodologies}

\subsection{MD simulation of Si using the environment-dependent interatomic potential}

In late 1990's, Bazant et al. [18, 19] and Justo et al. [20] developed an empirical potential for bulk Si, named environment-dependent interatomic potential (EDIP). This potential contains two and three-body interactions with theoretically motivated functional forms that give a better prediction of bulk defect and disordered phase of Si. The numerical parameters in the function are obtained by fitting a set of ab initio results. Work by Justo et al. [20] using the EDIP predicted structural and thermodynamic properties of liquid and amorphous Si that are in good agreement with experimental and ab initio results. 
The expression of the EDIP is a summation over the energy of each atom, and consists of twobody and three-body interaction terms [20],

$$
E_{i}=\sum_{j \neq i} V_{2}\left(R_{i j}, Z_{i}\right)+\sum_{j \neq i} \sum_{k \neq i, k>j} V_{3}\left(\vec{R}_{i j}, \vec{R}_{i k}, Z_{i}\right)
$$

$V_{2}\left(R_{i j}, Z_{i}\right)$ stands for the two-body interaction potential energy between atoms $i$ and $j$. The term $V_{3}\left(\vec{R}_{i j}, \vec{R}_{i k}, Z_{i}\right)$ represents the three-body potential energy among atoms $i, j$ and $k$ centered at $i$. The interaction of both types depends on the effective bond order which is defined as $Z_{i}=\sum_{m \neq i} f\left(R_{i m}\right)$. In the definition of the effective bond order, the function $f\left(R_{i m}\right)$ is called cutoff function, a measurement of the contribution of the neighboring atom $m$ to atom $i$ in terms of their distance $R_{i m}$. Definitions of the functions and values of parameters used in the EDIP can be found in work by Justo et al. [20].

In this work, the physical domain of interest is divided into computational cells based on the cutoff distance of the potential energy to improve the computational efficiency [21]. If the distance between two atoms is longer than the cutoff distance, both the two-body and three-body interactions are ignored and set to zero. The cutoff distance for Si has been given in earlier work [20]. In this work, the computation is organized using the cell structure and the linked-list method.

\subsection{NEMD for thermal conductivity calculation}

In this method, a temperature gradient is established across the material. The thermal conductivity is calculated based on the heat flux and the temperature gradient established in the 
sample. The first step of calculation is the equilibrium process to adjust the system to an expected temperature, which is $300 \mathrm{~K}$ in this work. In the equilibrium calculation, the scaling factor $S_{f}$ introduced by Berendsen et al. [22] is used to adjust the velocity

$$
S_{f}=\left[1+\frac{\Delta t}{\tau}\left(\frac{T_{0}}{T}-1\right)\right]^{1 / 2}
$$

where $\Delta t$ is the time step in the simulation. Although a short time step will ensure accurate and reliable calculation, it will make the total computational time not practical. In this work, we take the time step $2 \mathrm{fs}$ ( $1 \mathrm{fs}=10^{-15}$ second) based on our calculation experience and literatures. In Eq. (2), $\tau$ is the relaxation time, which is $2 \mathrm{ps}\left(1 \mathrm{ps}=10^{-12}\right.$ second $)$ in this work, $T$ is the current kinetic temperature, and $T_{0}(=300 \mathrm{~K})$ is the expected equilibrium temperature. This process takes $0.5 \mathrm{~ns}$ to ensure the system reaches equilibrium. The computational domain is schematically shown in Fig. 1. For the SWSNTs studied in this work, periodical boundary conditions are used in the $y$ (axial) direction. In the two other directions, free boundary conditions are used to give the sample certain freedom to move in space.

In the original NEMD method developed by Evans [23], the equation of motion for each particle has an external force term. In our work, we employ hot and cold domains in the material [24] and adjust the velocities of atoms in the domain to achieve local heating and cooling [25]. The equation used to obtain the thermal conductivity $(k)$ in NEMD is $k=q^{\prime \prime} /(\partial T / \partial x)$, where $\partial T / \partial x$ is the temperature gradient and $q^{\prime \prime}$ is the heat flux. Figure 1 shows how we establish a temperature gradient in the material. At each time step, a certain amount of energy is added in domain $A$ and the same amount of energy is taken away from domain $B$. Since the nanotube has periodical boundary conditions in the axial direction, two heat fluxes will flow along both positive and negative directions from domains $A$ to $B$. To establish the heat source and heat sink, 
the velocities of atoms in domains $A$ and $B$ are scaled with a factor $f$. During this velocity scaling, the momentum of atoms in domains $A$ and $B$ is conserved by only scaling the random movement of atoms, not their average velocity.

The size ( $\delta y)$ of domains $A$ and $B$ is chosen based on the length of the nanotube and ease of calculation. In this work, computation of the force between an atom and its neighbors is arranged by the cell structure and the linked-list method. The "cell size" used in this work is referred to the one used in the linked-list method. For SWSNTs longer than $0.5 \mu \mathrm{m}$, the typical values of $\delta y$ are 40 to 60 cell sizes (12 to $18 \mathrm{~nm}$ ). For short tubes, the size of domains is 5 to 10 cell sizes. In the NEMD technique, a sufficiently long computational time is needed to establish a stable temperature gradient in the material. In our work, the computational time $(t)$ is estimated by calculating the thermal diffusion time from domains $A$ to $B: t=(L / 2)^{2} / \alpha$, where $\alpha\left[=k /\left(\rho c_{p}\right)\right]$ and $L$ are the thermal diffusivity and length of the SWSNT, respectively. In the estimation, $\rho$ and $c_{p}$ take the bulk values and $k$ takes the value of a shorter SWSNT calculated previously. In this work, the computational time for the shortest ( $34 \mathrm{~nm}$ ) SWSNT is $0.5 \mathrm{~ns}$.

The temperature gradient in the tube is determined by linear fitting of the temperature distribution curve in the material. The thermal conductivity $(k)$ of the SWSNT can be calculated as

$$
k=-q^{\prime \prime} /\left(\partial T_{1} / \partial x-\partial T_{2} / \partial x\right)
$$

where $\partial T_{1} / \partial x$ and $\partial T_{2} / \partial x$ are the temperature gradients in the positive and negative $y$ directions along the tube. The temperature of the nanotube is calculated as 


$$
\frac{1}{2}\left\langle\sum_{i}^{N j} m v_{i}^{2}\right\rangle=\frac{3}{2} N_{j} k_{B} T_{M D}
$$

where $<>$ denotes the statistical average over the entire simulation (chosen to be much longer than the typical fluctuation time), $k_{B}$ is the Boltzmann's constant, and $N_{j}$ is the number of atoms in the layers for temperature calculation. 15 to 40 atomic layers (depending on the total length of the nanotube) along the $y$ direction are chosen to calculate one temperature point.

It is expected that the thermal conductivity of SWSNTs is directly related to how its crosssectional area is defined. In this work, an annulus consisting of atoms in the SWSNTs is defined as the cross-sectional area. The annulus thickness takes two times the covalent radius of Si atoms, which is $1.175 \AA$ [26].

In addition to thermal conductivity, we also study the specific heat of SWSNTs to attain the idea about how their unique structure affects their capability of storing thermal energy. The method used for specific heat calculation is the same as that employed in work by Wang and $\mathrm{Xu}$ [27]. In the calculation, a certain amount of energy $\Delta E$ is added to the system. This energy addition is achieved through a large number of time steps $(10,000)$ to minimize the thermal and mechanical disturbance introduced to the system. Then the system is simulated for $0.3 \mathrm{~ns}$ to reach thermal equilibrium. The specific heat is calculated as

$$
c_{p}=\Delta E /\left\lfloor m\left(T_{\text {final }}-T_{\text {initial }}\right)\right\rfloor
$$

where $m$ is the mass of the entire tube, $T_{\text {initial }}$ is the temperature before adding the energy, and $T_{\text {final }}$ is the temperature after energy addition. In our simulation, the temperature is calculated every 2 ps. $T_{\text {initial }}$ and $T_{\text {final }}$ are obtained by averaging the temperatures of the last $120 \mathrm{ps.}$ 


\section{Results and discussion}

In this work, SWSNTs with three different structures are studied. Figure 2 shows the SWSNT structure and the bond length. Table 1 lists the length and number of atoms simulated for each type of nanotubes in this work. In our thermal conductivity study (3.2), comparison is also made with Si films of different thicknesses. The thickness and number of atoms for the simulated Si films are also summarized in Table 1 . The initial temperature of the nanotube is set to $300 \mathrm{~K}$, and the time step takes $2 \mathrm{fs}$. The system is first calculated for $500 \mathrm{ps}$ to reach the thermal equilibrium state. Then calculation is carried out to establish a steady temperature gradient in the material. This calculation takes 0.5 to $8 \mathrm{~ns}$, depending on the length of the nanotube as described in section 2.2.

\subsection{Structure of SWSNTS}

In order to have a better understanding of the structure of the three kinds of SWSNTs, the radial distribution function $g\left(r_{i}\right)$ and the atomic energy distribution are calculated and compared with those of bulk Si. Figure 3 shows the radial distribution function with $r$ up to $8 a . a$ is a parameter in the EDIP potential, and takes 3.1213820 ̊. The position of the first peak for SWSNTs shows that the distance between an atom and its nearest neighbor is $2.34 \AA$, close to the bond length of c-Si at $300 \mathrm{~K}(2.35 \AA)$. This result suggests that SWSNTs have bond length almost the same as that of bulk c-Si. The second peak of both SWSNTs and bulk Si represents the distance to the second nearest neighboring atom. For SWSNTs, the distance between an atom and its second nearest neighboring atom is shown in Fig. $2 \mathrm{~b}$. The ab initio prediction of the second nearest neighboring distance is $3.384 \AA(1.084 a), 3.386 \AA(1.085 a)$, and $3.360 \AA(1.076 a)$ for 
rectangular, pentagonal, and hexagonal SWSNTs, respectively. Figure 3 indicates that using the bond length provided by $a b$ initio calculations, the classical MD simulation based on the EDIP works well to preserve the structure of SWSNTs. The number of the second nearest neighboring atoms for an atom in pentagonal and hexagonal SWSNTs is 4, and 5 for rectangular SWSNTs. In our MD simulation, the outside of the SWSNT has no constraint. Some weak stress due to the initial configuration will twist the tube in space a little bit. Our careful observation of the tube structure confirms this point. This twist will affect the tube structure to a certain extent and make the second peak of SWSNTs lower and wider. Since the equilibrium angle of bulk Si is $109^{\circ}$, the second peak of bulk Si is shifted to a further distance (compared with SWSNTs) which is about $3.85 \AA(1.233 a)$.

The atomic energy distribution is related to the bond length and the physical properties of the material. It also indicates the probability of finding an atom at a certain energy level. In our work, after the system reaches thermal equilibrium, the kinetic energy distribution is calculated by counting the number of atoms in a certain energy range. After normalizing the distribution, we confirm that the kinetic energy closely follows the Maxwellian distribution. Therefore, only the potential energy distribution is discussed in detail, especially the two-body potential which plays a major role in the overall energy distribution. Equation (1) reveals that $Z$ and $r$ play major roles in determining the two-body potential energy. The sharpness of the first peak in the radial distribution of bulk Si (shown in Fig. 3) demonstrates that the first nearest neighboring distance is quiet uniform. Therefore, the major factor affecting the two-body potential is the value of $Z$. In this work, the value of $Z$ is calculated for both SWSNTs and bulk c-Si. 
Figure 4(a) shows the two-body potential energy distribution for SWSNTs and bulk Si. It can be seen that bulk Si has a sharp peak at $-2.33 \mathrm{eV}$. The energy of the two-body potential of two $\mathrm{Si}$ atoms at equilibrium for bulk $\mathrm{Si}$ should be $-1.16 \mathrm{eV}$ for $Z$ equal to 4 [20], meaning the two-body potential energy for bulk Si will be $-2.32 \mathrm{eV}$ since each atom has four neighboring atoms. This value is close to the energy of the first peak for bulk Si shown in Fig. 4. The two small peaks of energy distribution in Fig. 4(a) for bulk $\mathrm{Si}$ are related to the atoms at the boundary whose $Z$ values are 3 or 2 . In order to give a clearer view of the sharp peak energy of bulk $\mathrm{Si}$, the first peak of the atomic energy distribution for bulk $\mathrm{Si}$ is detailed in the inset. It can be seen that the most prominent range for the two-body potential energy is -2.30 to $-2.325 \mathrm{eV}$.

As we stated before, SWSNTs were twisted a little bit during the calculation. This makes the distance between atoms reach the range where the cutoff function follows the exponential form in the EDIP, leading to non-integer $Z$ values. Our calculation shows that the $Z$ of SWSNTs largely falls in the range of 4.8 5.0, leading to a smoother and wider distribution of the two-body potential energy than bulk $\mathrm{Si}$ as shown in Fig. 4. In addition, the range of bond length distribution of SWSNTs is also an important reason for the smoother and wider distribution of the two-body potential energy. When $Z$ is equal to 5 , the theoretical two-body potential energy should be about $-0.9 \mathrm{eV}[20]$, meaning that the two-body potential energy for SWSNTs will be around $-2.25 \mathrm{eV}$ since SWSNTs have 5 neighboring atoms within the cutoff distance. The calculated peak of the two-body potential energy for SWSNTs is around $-2.30 \mathrm{eV}$, close to the predicted value of $-2.25 \mathrm{eV}$ [20]. The value of $Z$ reflects the electronic properties of materials. According to work by Justo et al. [20], $Z$ larger than 4 means metallic bonds among atoms. When $Z$ is less than or equal to 4 , the atomic bonds tend to be covalent. The value of the 
effective bond order $Z$ obtained in our work for c-Si (which is 4) reflects the semiconducting nature of c-Si. On the other hand, the $Z$ for SWSNTs is from 4.8 to 5.0 based on our calculation. This agrees with the ab initio prediction that metallic Si structures have a bond order larger than $4[3]$.

Figure 4(b) shows the overall energy (including kinetic, two-body potential, and three-body potential energies) distribution of bulk Si and SWSNTs. The overall energy distribution for bulk Si has a wider and smoother distribution than the two-body potential energy. Our calculation shows that the three-body potential energy for bulk Si has a sharp peak close to $0 \mathrm{eV}$, agreeing with the result by Justo et al. [20]. In comparison with the two-body potential energy, the peak of the total energy does not shift too much because both the three-body potential and kinetic energies are relatively small. In contrast to the small value of the three-body potential energy for c-Si, our calculation concludes that the three-body potential energy of SWSNTs is around 0.23 $\mathrm{eV}$. The reason is that the angle of the three-body potential in SWSNTs is different from that of c-Si, causing the three-body potential energy of SWSNTs to shift further from zero. Hence, the peaks of the total energy distribution of SWSNTs have a significant shift to right in comparison with their two-body potential energy distribution.

\subsection{Thermal conductivity and specific heat of SWSNTs}

In NEMD for thermal conductivity calculation, a stable temperature gradient has to be established in the material. Figure 5 shows a typical temperature distribution in a $485.8 \mathrm{~nm}$ long pentagonal SWSNT. It is evident that a sound linear temperature distribution is established in the material. In our work, since the number of atoms used for temperature calculation is small due to 
the small size of the tube, a large temperature differential between domains $A$ and $B$ (refer to Fig. 1) is needed to suppress the statistical uncertainty. The temperature differential between domains $A$ and $B$ is about $150 \mathrm{~K}$, depending on the size of SWSNTs under study. In the area close to domains $A$ and $B$, abrupt temperature drops (as shown in Fig. 5) usually exist due to the heating and cooling effects in these domains. These abrupt temperature drops are out of consideration in final data reduction to obtain a sound linear temperature distribution. Also shown in Fig. 5 is the linear fitting of the temperature distribution. It provides the temperature gradient used in Eq. (3) to determine the thermal conductivity of SWSNTs.

In this work, SWSNTs with different lengths are studied to explore their thermal conductivity. Table 1 shows the length of SWSNTs studied in this work as well as the number of atoms in the tubes. In addition, the thermal conductivity $\left(k_{\text {eff }}\right)$ in the thickness $(L)$ direction for Si thin films is also calculated and compared with that of SWSNTs. The thickness of the films is listed in Table 1 as well. It is found the $k_{\text {eff }} L$ relationship closely follows that obtained by LBM [28]

$$
\frac{k_{e f f}}{k_{b}}=\left(1+2 \frac{l}{L}\right)^{-1}
$$

where $l$ is the mean free path of phonons in bulk Si.

To obtain the idea about how the structure of SWSNTs affects their thermal conductivity, we plot out the thermal conductivity of three different types of SWSNTs in one plot for purpose of comparison (Fig. 6). Also shown in Fig. 6 is the error bar for uncertainty analysis. This uncertainty is evaluated by dividing the overall temperature differential across the sample with the standard deviation in linear fitting of the temperature gradient. Our study of the linear fitting for different samples reveals that the uncertainty is well within $15 \%$. For Si thin films, the 
uncertainty for thermal conductivity calculation is smaller (10\%). It is observed in Fig. 6 that the thermal conductivity of SWSNTs is strongly dependent on their length. When the length reaches about $1 \mu \mathrm{m}$, the thermal conductivity reaches a level of about $30 \mathrm{~W} / \mathrm{m} \cdot \mathrm{K}$, which is still significantly less than the thermal conductivity of bulk c-Si at $300 \mathrm{~K}(148 \mathrm{~W} / \mathrm{m} \cdot \mathrm{K})$ [29]. On the other hand, the thermal conductivity of SWSNTs shown in Fig. 6 is much larger than that of Si nanowires (from 1 to $4 \mathrm{~W} / \mathrm{m} \cdot \mathrm{K}$ ) as reported in work by Chen and Volz [9]. This is attributed to the entirely different atomic structure of SWSNTs from Si nanowires. In Si nanowires, strong phonon boundary scattering occurs on the wire surface, leading to a much reduced thermal conductivity. In SWSNTs, the wall of the tube is a single atomic layer, and the phonon movement is largely constrained in the axial direction. Therefore, very little surface phonon scattering is permitted.

Figure 6 shows that SWSNTs with hexagonal cross-sections have the lowest thermal conductivity, and the pentagonal SWSNTs have the highest one. It is clear that the thermal conductivity of Si thin films is very different from that of SWSNTs. The thermal conductivity of Si thin films increases quickly with the thickness and reaches $116.3 \mathrm{~W} / \mathrm{m} \cdot \mathrm{K}$ at the thickness of $300 \mathrm{~nm}$. This thermal conductivity is only 17 percent less than the bulk value. However, with the similar characteristic size, the thermal conductivity of SWSNTs is $14.1 \mathrm{~W} / \mathrm{m} \cdot \mathrm{K}$ for rectangular SWSNTs $(263.2 \mathrm{~nm}), 19.8 \mathrm{~W} / \mathrm{m} \cdot \mathrm{K}$ for pentagonal SWSNTs $(272.00 \mathrm{~nm})$, and $8.0 \mathrm{~W} / \mathrm{m} \cdot \mathrm{K}$ for hexagonal SWSNTs $(250.95 \mathrm{~nm})$. The striking difference between Si thin films and SWSNTs about how the thermal conductivity changes with their thickness/length suggests that the 1D structure of SWSNTs leads to a stronger phonon boundary scattering effect at the tube end. 
Recently, the relationship between the one dimensional anomalous heat conduction and the material dimension has been investigated by $\mathrm{Li}$ and Wang [30]. They proposed a thermal conductivity ( $k$ )-length $(L)$ relationship of $k=c L^{\beta}$ with $\beta=2-2 / \alpha(0<\alpha \leq 2)$. For normal diffusion $\alpha$ is equal to 1 , and for superdiffusion $\alpha$ is greater than 1 , meaning a divergent thermal conductivity $(\beta>0)$. Subdiffusion $(\alpha<1)$ gives a convergent thermal conductivity $(\beta<0)$. It is reported by Wang and $\mathrm{Li}[31]$ that in many $1 \mathrm{D}$ chains with momentum conservation, $1 / 3$ and 2/5 are the generic exponent for the divergent thermal conductivity. The coupling between the transverse motion of the atoms with the longitudinal motion will lead to a thermal conductivity diverging against the system size with $1 / 3$ power law. On the other hand, in the weak coupling regime, a $2 / 5$ power law is observed. One new work [32] showed that a finite thermal conductivity can be reached only when disorder exists in the 1D chain. Furthermore, Zhang and Li [33] reported the value of the exponent for $(5,5)$ and $(10,10)$ single-wall CNTs (SWCNTs) at $300 \mathrm{~K}$ and $800 \mathrm{~K}$. Their results showed that at $300 \mathrm{~K}$ the $(5,5) \mathrm{SWCNT}$ follows the law of $2 / 5$ and the exponent of $(10,10)$ SWCNT is 0.36 . Recently, the thermal conductivity of $(10,0)$ SWCNTs was suggested to be proportional to $L^{1 / 2}$ [34]. In work by Maruyama [2], the thermal conductivity of $(5,5)$ SWCNTs is divergent with a power of 0.32 . Maruyama pointed out that the structure will strongly affect the power. The $\beta$ exponents for $(5,5),(8,8)$ and $(10,10)$ are quite different, where the $(5,5)$ SWCNT has the biggest value and the $(10,10)$ has the smallest one. Our results indicate that the pentagonal SWSNTs follow the curve with a power of $2 / 5$. On the other hand, the thermal conductivity of hexagonal and rectangular SWSNTs is proportional to $L^{0.68}$ and $L^{0.62}$, respectively. The difference in the power probably is due to the cross-section structures of the SWSNTs which favor different coupling between the transverse and longitudinal movements of atoms. 
For specific heat, we first calculate the value of bulk c-Si at $300 \mathrm{~K}$ to explore the validity of the developed MD program. The sample of interest measures $6.52 \mathrm{~nm}$ in the $x, y$, and $z$ directions, and consists of 13,824 atoms. The calculated specific heat for c-Si is $830 \mathrm{~J} / \mathrm{K} \cdot \mathrm{kg}$, a little larger than the experimental result of $712 \mathrm{~J} / \mathrm{K} \cdot \mathrm{kg}$ [29]. In order to check the influence of the tube length on the calculated specific heat, three pentagonal SWSNTs samples have been studied, which are 136.0, 233.1 and $485.8 \mathrm{~nm}$ long. Table 2 shows the calculated specific heat for c-Si and SWSNTs. It is clear that the largest difference between the three pentagonal SWSNTs is about $5 \%$. As can be seen from Table 2, the specific heat of SWSNTs is a little larger than that of bulk

c-Si. Rectangular SWSNTs have the lowest specific heat, i.e. close to that of bulk Si, and pentagonal and hexagonal SWSNTs have similar specific heats. The uncertainty of specific heat calculation is estimated using the root mean square (RMS) of equilibrium temperature vibration as $R M S / \Delta T$, where $\Delta T$ is the average temperature increase after the system is heated. The uncertainty for specific heat calculation is estimated to be around $5 \%$.

\section{Conclusion}

In this work, MD simulation was conducted to explore the structure and thermophysical properties of SWSNTs of different cross-section structures and lengths. The structure of SWSNTs led to a wider range of energy distribution than c-Si. It was found that the unique structure of SWSNTs resulted in a bond order in the range of 4.8 5. This range agreed with the ab initio prediction that metallic Si structures have a bond order larger than 4 . The calculation showed that the thermal conductivity of SWSNTs was much smaller than that of bulk Si. There was a significant difference between SWSNTs and c-Si films on how their length/thickness 
affected the thermal conductivity. This striking difference probably is due to the strong longitudinal movement of atoms in SWSNTs and weak coupling between the transverse and longitudinal movements. Out of the three types of SWSNTs under study, pentagonal SWSNTs had the highest thermal conductivity while hexagonal SWSNTs had the lowest one. Our study showed that the specific heat of SWSNTs was a little larger than that of bulk c-Si. Pentagonal and hexagonal SWSNTs had close specific heats which were a little larger than that of rectangular SWSNTs. 


\section{Acknowledgements}

Support for this work from NSF (CMS: 0457471), Nebraska Research Initiative, and Layman Award of the University of Nebraska-Lincoln is gratefully acknowledged. 


\section{References}

1. S. Iijima and T. Ichihashi, Nature 363 (1993) 603.

2. S. Maruyama, Microscale Thermophys. Eng. 7 (2003) 41.

3. J. Bai, X. C. Zeng, H. Tanaka, and J. Y. Zeng, Proc. Natl. Acad. Sci. 101 (2004) 2664.

4. Y. Zhen, C. Rivas, R. Lake, K. Alam, T. B. Boykin, and G. Klimeck, IEEE Transactions On Electron Devices 52 (2005) 1097.

5. J. Hone, M. Whitney, C. Piskoti, and A. Zettl, Phys. Rev. B 59 (1999) 2514.

6. D. J. Yang, Q. Zhang, G. Chen, S. F. Yoon, J. Ahn, S. G. Wang, Q. Zhou, Q. Wang, and J. Q. Li, Phys. Rev. B 66 (2002) 165440.

7. X. W. Wang, Z. R. Zhong, and J. Xu, J. Appl. Phys. 97 (2004) 064302.

8. M. A. Osman and D. Srivastava, Nanotechnology 12 (2001) 21.

9. S. G. Volz and G. Chen, Appl. Phys. Lett. 75 (1999) 2056.

10. S. G. Volz and G. Chen, Phys. Rev. B 61 (2000) 2651.

11. C. J. Gomes, M. Madrid and C. H. Amon, ASME International Mechanical Engineering Congress and Research Exposition (2003) 42352.

12. X. L. Feng, Microscale Thermophys. Eng. 7 (2003) 153.

13. X. W. Wang and C. Lawrence, ASME Heat Transfer/Fluids Engineering Summer Conference, paper \#: HT-FED2004-56725 (2004).

14. Y. H. Lee, R. Biswas, C. M. Soukoulis, C. T. Wang and K. M. Ho, Phys. Rev. B 43 (1991) 6573.

15. R. D. Mountain and R. A. MacDonald, Phys. Rev. B 28 (1983) 3022.

16. P. Jund and R. Jullien, Phys. Rev. B 59 (1999) 13707.

17. P. K. Schelling, S. R. Phillpot and P. Keblinski, Phys. Rev. B 65 (2002) 144306.

18. M. Z. Bazant and E. Kaxiras, Phys. Rev. Lett. 77 (1996) 4370. 
19. M. Z. Bazant, E. Kaxiras and J. F. Justo, Phys. Rev. B 56 (1997) 8542.

20. J. F. Justo, M. Z. Bazant, E. Kaxiras, V. V. Bulatov and S. Yip, Phys. Rev. B 58 (1998) 2539.

21. M. P. Allen and D. J. Tildesley, Computer Simulation of Liquids (Clarendon Press, Oxford, 1987).

22. H. J. C. Berendsen, J. P. M. Postma, W. F. van Gunsteren, A. DiNola, and J. R. Haak, J. Chem. Phys. 81 (1984) 3684.

23. D. J. Evans, Phys. Lett. 91A (1982) 457.

24. P. Jund and R. Jullien, Phys. Rev. B 59 (1999) 13707.

25. M. Atsuhiko and M. Toyonori, Phys. Rev. E 52 (1995) 234.

26. http://serve.me.nus.edu.sg/nanomachining/wafer_materials.htm.

27. X. Wang and X. Xu, J. of Heat Transfer 124 (2002) 265.

28. J. Xu, Master Thesis, University of Nebraska-Lincoln, 2004.

29. F. P. Incropera and D. P. DeWitt, Fundamentals of Heat and Mass Transfer, 5th Ed. (John Wiley \& Sons, New York, 2002).

30. B. Li and J. Wang, Phys. Rev, Lett. 91 (2003) 044301.

31. J. Wang and B. Li, Phys. Rev. Lett. 92 (2004) 074302.

32. B. Li, L. Wang and B. Hu, Phys. Rev. Lett. 88 (2002) 223901.

33. G. Zhang and B. Li, J Chem. Phys. 123 (2005) 114714.

34. N. Mingo and D. A. Broido, Nano Lett. 5 (2005) 1221. 


\section{List of Tables and Figures}

Table 1 Thickness (in $\mathrm{nm}$ ) of Si films and length (in $\mathrm{nm}$ ) of SWSNTs simulated in this work. The number of atoms in the sample is indicated in the parentheses.

Table 2 Specific heat of bulk Si and SWSNTs.

Figure 1 Schematic of the computational domain. Arrows in the tube represent the heat flux direction.

Figure 2 Schematic of the structure of (a) rectangular, (b) pentagonal, and (c) hexagonal SWSNTs.

Figure 3 The radial distribution function of SWSNTs versus that of bulk c-Si.

Figure 4 Energy distribution of atoms in SWSNTs in comparison with that in bulk Si: (a) two-body potential energy, and (b) total atomic energy. The inset in figure (a) is a close view of the two-body potential energy distribution for bulk Si around the peak. A finer resolution is used for calculating the energy distribution shown in the inset.

Figure 5 Temperature distribution in a pentagonal SWSNT $485.8 \mathrm{~nm}$ long. The solid line is the linear fitting of the temperature distribution.

Figure 6 Variation of thermal conductivity against length for SWSNTs in comparison with that for bulk Si. 
Table 1: Thickness (nm) of Si films and length (nm) of SWSNTs simulated in this work. The number of atoms in the sample is indicated in the parentheses.

\begin{tabular}{c|c|c|c}
\hline Si thin film & Rectangular SWSNTs & Pentagonal SWSNTs & Hexagonal SWSNTs \\
\hline $10(16,000)$ & $75.2(1,240)$ & $68(1,400)$ & $74.1(1,860)$ \\
\hline $32.5(48,000)$ & $148.4(2,500)$ & $136(2,800)$ & $149.4(3,750)$ \\
\hline $53.7(79,200)$ & $263.2(4,400)$ & $272(5,600)$ & $286.8(7,200)$ \\
\hline $70.1(104,800)$ & $526.5(8,800)$ & $485.8(10,000)$ & $501.9(12,600)$ \\
\hline $100(148,800)$ & $1052.9(17,600)$ & $971.6(20,000)$ & $1003.8(25,200)$ \\
\hline $200(297,600)$ & $1579.4(26,400)$ & $1457.4(30,000)$ & $1505.7(37,800)$ \\
\hline $300(446,400)$ & $2105.8(35,200)$ & $1943.2(40,000)$ & $2007.6(50,400)$ \\
\hline
\end{tabular}


Table 2. Specific heat of bulk Si and SWSNTs.

\begin{tabular}{c|c}
\hline Materials & Specific heat $(\mathrm{J} / \mathrm{K} \cdot \mathrm{kg})$ \\
\hline Bulk c-Si (experiment) & 712 \\
\hline Bulk c-Si (MD) & 830 \\
\hline Rectangular SWSNT $(526 \mathrm{~nm})$ & 856 \\
\hline Pentagonal SWSNT $(136 \mathrm{~nm})$ & 937 \\
\hline Pentagonal SWSNT $(233 \mathrm{~nm})$ & 906 \\
\hline Pentagonal SWSNT $(486 \mathrm{~nm})$ & 891 \\
\hline Hexagonal SWSNT $(502 \mathrm{~nm})$ & 896 \\
\hline
\end{tabular}




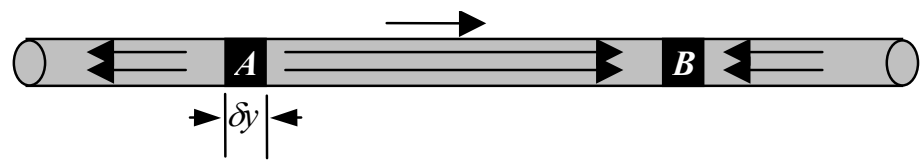

Figure 1. Schematic of the computational domain. Arrows in the tube represent the heat flux direction. 
The second nearest

neighboring distance

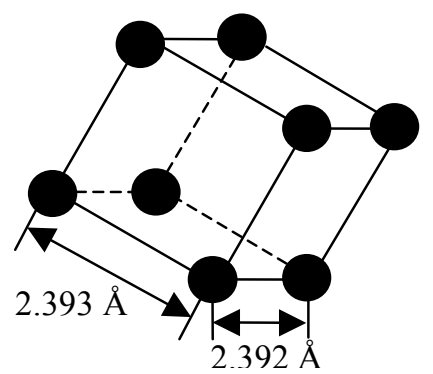

(a)

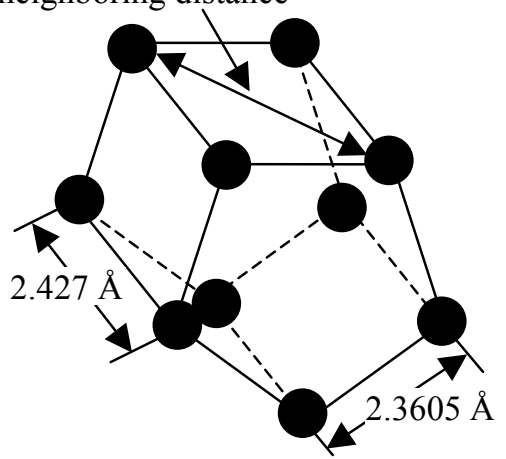

(b)

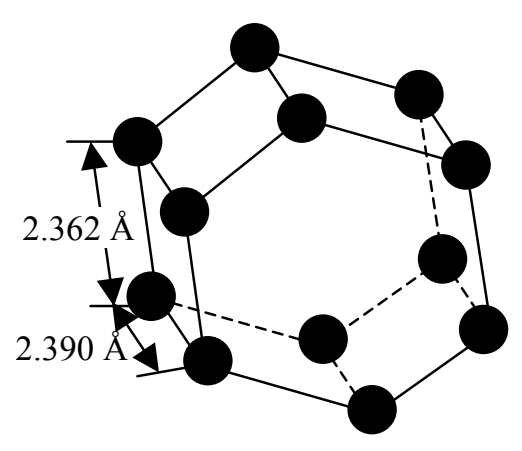

(c)

Figure 2. Schematic of the structure of (a) rectangular, (b) pentagonal, and (c) hexagonal SWSNTs. 


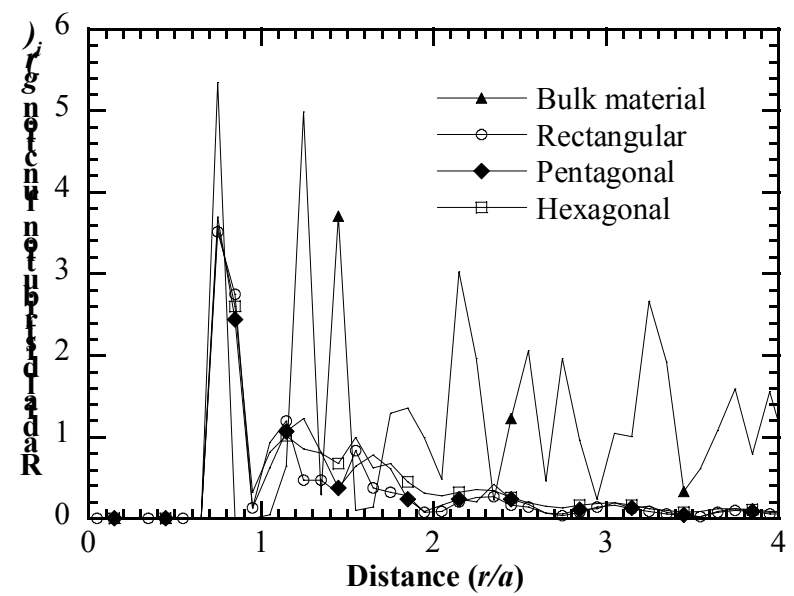

Figure 3. The radial distribution function of SWSNTs versus that of bulk c-Si. 

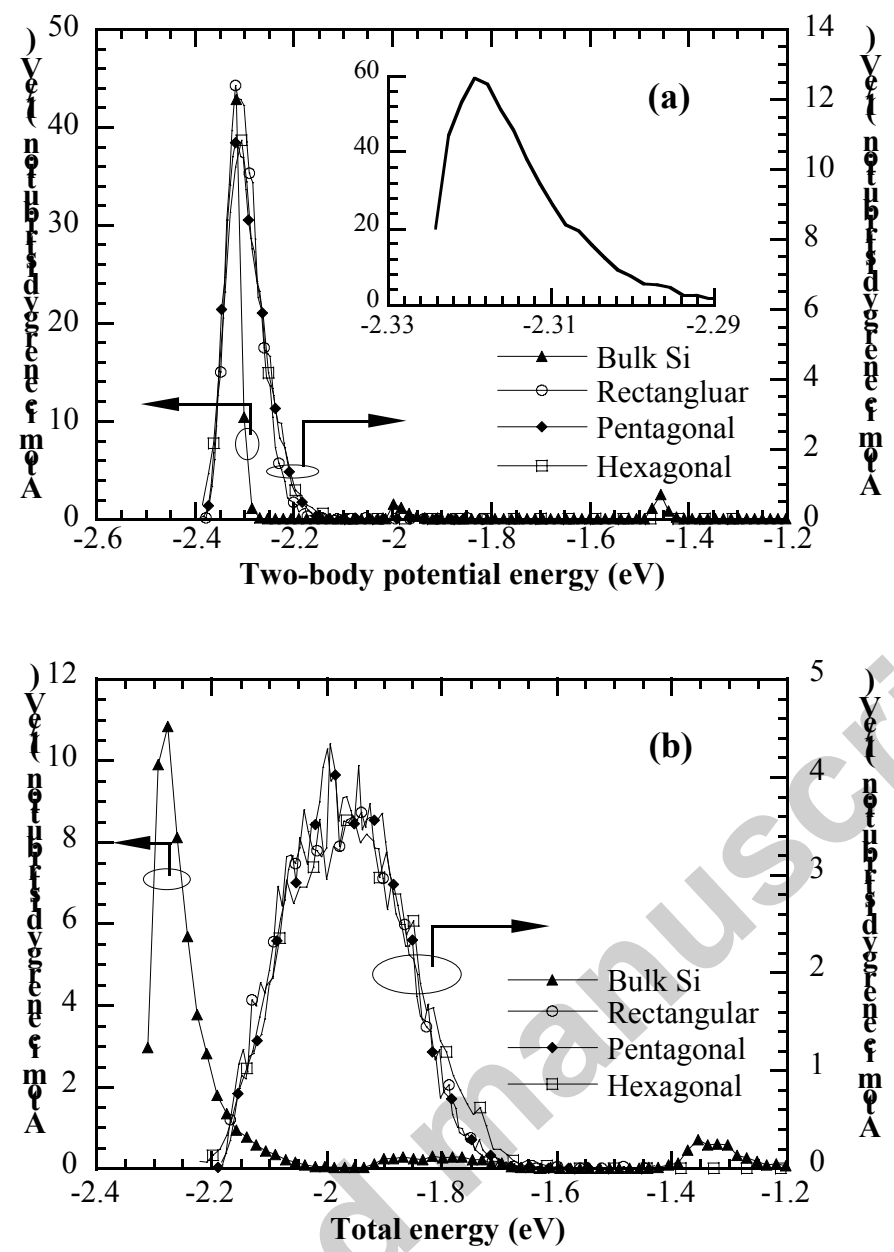

Figure 4. Energy distribution of atoms in SWSNTs in comparison with that in bulk Si: (a) twobody potential energy, and (b) total atomic energy. The inset in figure (a) is a close view of the two-body potential energy distribution for bulk Si around the peak. A finer resolution is used for calculating the energy distribution shown in the inset. 


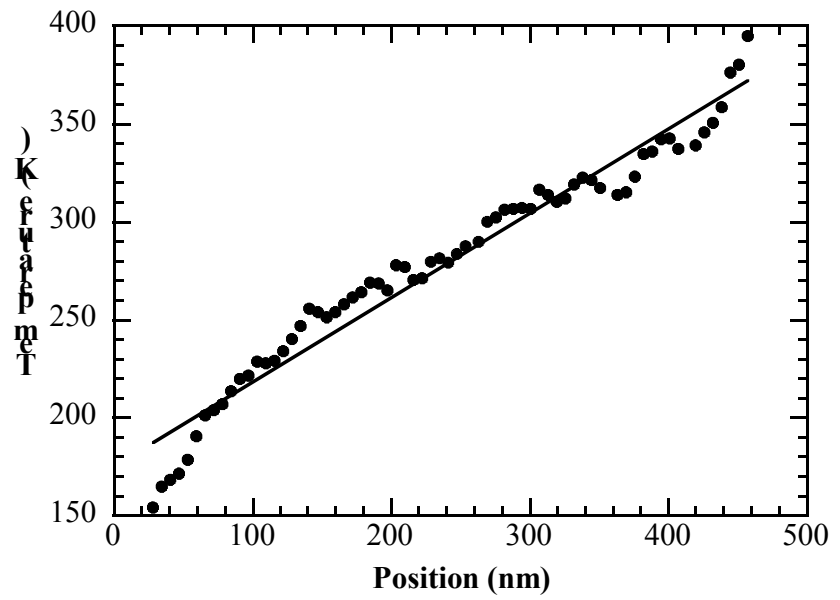

Figure 5. Temperature distribution in a pentagonal SWSNT $485.8 \mathrm{~nm}$ long. The solid line is the linear fitting of the temperature distribution. 


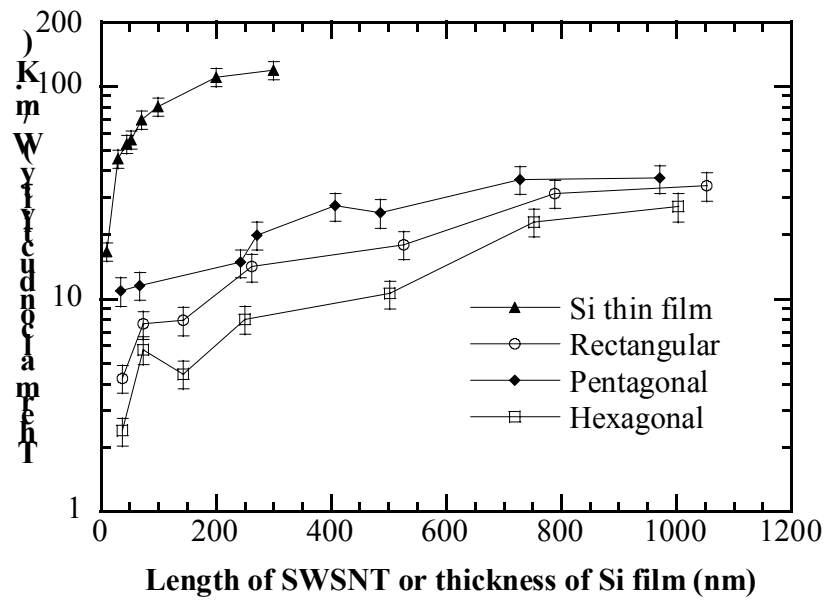

Figure 6. Variation of thermal conductivity against length for SWSNTs in comparison with that for bulk Si: $148 \mathrm{~W} / \mathrm{m} \cdot \mathrm{K}[29]$. 